In conclusion, half-fenestrated stent grafting was feasible and effective as a less invasive treatment for mycotic aneurysm when infection was medically controlled. Visceral blood supply was spared by using the technique we applied.

\section{References}

1. Ihaya A, Chiba Y, Kimura T, Morioka K, Uesaka T. Surgical outcome of infectious aneurysm of the abdominal aorta with or without SIRS. Cardiovasc Surg. 2001;9:436-40.
2. Madhavn P, McDonnell CO, Dowd MO, Sultan SAH, Doyle M, Colgan MP, et al. Suprarenal mycotic aneurysm exclusion using a stent with a partial autologous covering. J Endovasc Ther. 2000;7:404-9.

3. McGraw JK, Patzik SB, Gale SS, Dodd JT, Boorstein JM. Autogenous vein-covered stent for the endovascular management of a superior mesenteric artery pseudoaneurysm. J Vasc Interv Radiol. 1998;9:779-82.

4. Cowan S, Kahn MB, Bonn J, Becker GJ, DiMuzio P, Leichter R, et al. Superior mesenteric artery pseudoaneurysm successfully treated with polytetrafluoroethylene covered stent. J Vasc Surg. 2002;35: 805-7.

5. Kinney EV, Kaebnick HW, Mitchell RA, Jung MT. Repair of mycotic paravisceral aneurysm with a fenestrated stent-graft. J Endovasc Ther. 2000;7:192-7.

\title{
The gut-first approach for repair of Crawford extent III thoracoabdominal aortic aneurysms
}

\author{
Matthias Karck, MD, Christian Hagl, MD, Klaus Kallenbach, MD, and Axel Haverich, MD, Hannover, Germany
}

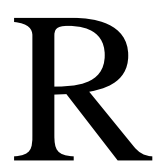

eplacement of thoracoabdominal aortic aneurysms requires reattachment of the visceral and renal artery origins to one or more side openings in the vascular graft. ${ }^{1,2}$ In Crawford extent III and IV aneurysms reimplantation and restoration of blood flow through the intestinal and renal arteries is followed by replacement of the infrarenal axis down to the aortic bifurcation or even below, with a crossclamp on the vascular graft. We describe a modification of this classical approach by interpositioning a vascular graft between the thoracic aorta and an isolated tissue patch containing the origins of the celiac, mesenteric, and left renal arteries in an end-to-end fashion. In a second step repair of the downstream aorta can be accomplished in a crossclamp-free operating field.

\section{Clinical Summary}

A 68-year-old man presented with a Crawford extent III aneurysm and chronic aortic dissection type B (Figure 1). Access to the thoracoabdominal aorta was provided by a thoracotomy in the seventh interspace, transection of the costal margin, circular division of the diaphragm, and subsequent retroperitoneal preparation. After systemic heparinization, femorofemoral cardiopulmonary bypass was initiated for maintenance of distal perfusion and in-

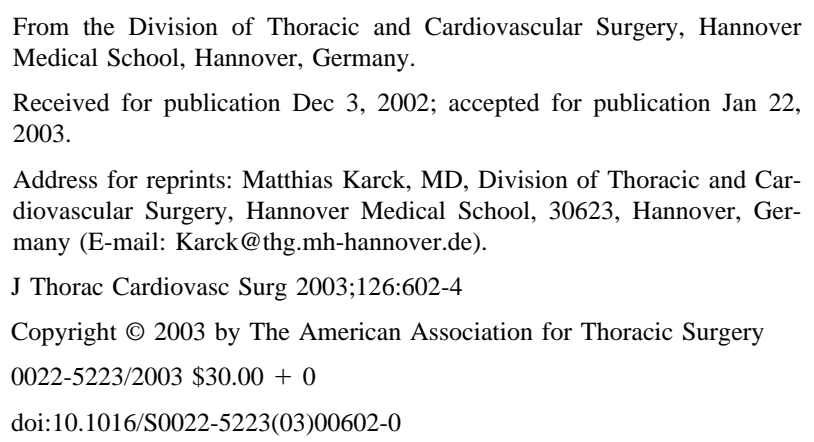

duction of mild hypothermia at $34^{\circ} \mathrm{C}$. The thoracic aorta was transected between occlusion clamps placed at the level of T4 and T7. The dissected layers of the aortic wall were reconstructed with gelatin resorcin formaldehyde glue before an anastomosis was carried out between a collagen-impregnated woven vascular graft of $32 \mathrm{~mm}$ in diameter (Hemashield, Boston Scientific) and the proximal descending aorta. Then the distal clamp was moved down to the level of the diaphragm before the aorta was incised longitudinally. Two intercostal arteries arising from T8 and T9 were reattached to the graft and reperfused after translocation of the proximal clamp. With release of the distal clamp, extracorporeal perfusion was discontinued. The aneurysm was opened longitudinally posterior to the left renal artery to its distal termination at the aortic bifurcation. The septum separating the true and false lumens was resected. The nondissected visceral and left renal artery origins were excised from the aortic wall as a single tissue patch and anastomosed end to end to the oblique distal end of the vascular graft (Figure 1, right). Then the crossclamp on the vascular graft was released, and antegrade flow into the visceral and left renal arteries was reestablished. The right renal artery was subsequently reattached to the prosthesis by means of interposition of a separate, small-diameter $(8 \mathrm{~mm})$ woven vascular graft. The last step comprised a vascular graft-to-aortic bifurcation anastomosis and a final graft-to-graft anastomosis in an end-to-side fashion, thereby completing the repair. Extracorporeal circulation was reestablished for rewarming. Remnants of the aneurysmal wall were loosely wrapped around the aortic graft by using a running suture before the operation was concluded as usual. The patient was extubated 2 days postoperatively, and the further postoperative course was uneventful.

\section{Discussion}

Thoracoabdominal aortic replacement in patients with chronic dissection requires a thorough preparation of one or more vascular patches carrying visceral and renal arteries to be reimplanted into a vascular graft. ${ }^{1,2}$ At first, this implies resection of the dissecting 

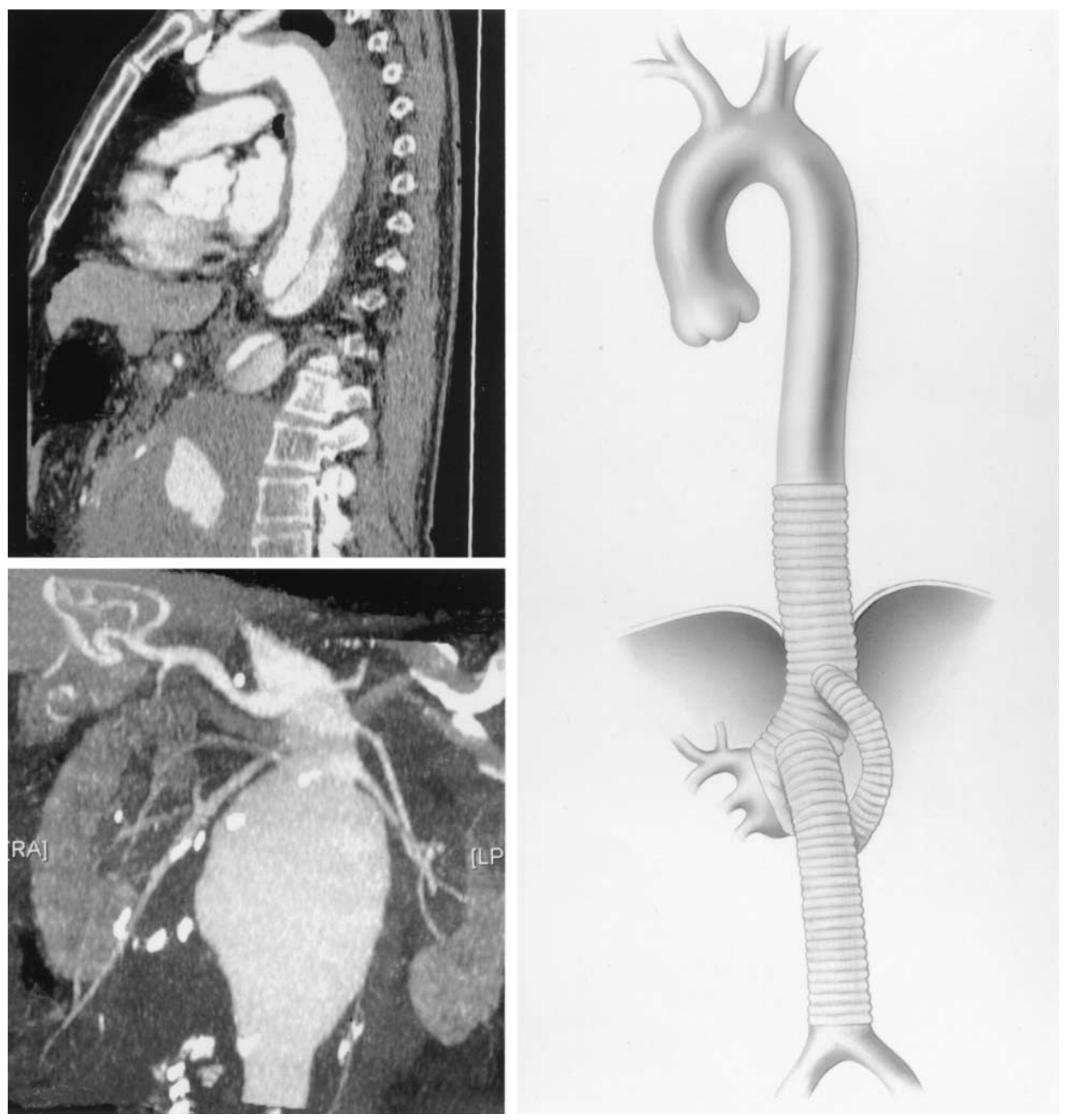

Figure 1. Upper left panel, Sagittal section: chronic aortic dissection type B. The false lumen is perfused in the lower third of the thoracic aorta, whereas the proximal segment is thrombosed. Severe kinking of the distal thoracic aorta is seen. Lower left panel, Oblique section: aneurysmatic dilatation of the infrarenal aorta. Right panel, Gut-first approach to repair of Crawford extent III thoracoabdominal aortic aneurysms. The vascular prosthesis for replacement of the thoracic aorta is anastomosed in an end-to-end fashion to a completely isolated vascular patch carrying the ostia of the celiac, superior mesenteric, and left renal arteries. The right renal artery is reattached with a small-diameter vascular graft. With all visceral arteries reperfused, the distal end of the replacement at the level of the aortic bifurcation can be accomplished in a clamp-free operating field. A final graft-to-graft end-to-side anastomosis completes the repair.

membrane separating the true and false aortic lumens. In many patients with the celiac, superior mesenteric, and left renal arteries arising from the true lumen, this line of separation might come very close to the respective ostia. In these cases it is advisable to isolate the vascular patch circumferentially to obtain complete control over the adjacent aortic adventitia for later inclusion into the suture line.

These patients are possible candidates for the technical modification described here. Even though a variable number of intercostal arteries are reimplanted into the vascular graft before, this variant might be named the gut-first approach in the style of the arch-first technique described by Griepp and Ergin. ${ }^{3}$ Complete isolation of this vascular patch represents a helpful precondition to perform a quick and easy end-to-end anastomosis to the distal end of the vascular graft instead of a sometimes cumbersome end-to-side anastomosis. Depending on the individual anatomy, this maneuver still allows subsequent direct reattachment of the right renal artery to the graft or, as in the case presented here, interposition of a small-diameter tube graft. With all visceral arteries reperfused, the distal end of the replacement at the level of the aortic bifurcation or below can be accomplished in a clamp-free operating field, which helps to improve exposure in a sometimes deep operating field. A final graft-to-graft end-to-side anastomosis completes this type of repair, which might facilitate surgical treatment of patients with Crawford extent III aneurysms. 


\section{References}

1. Coselli JS, LeMaire SA. Surgical techniques: thoracoabdominal aorta. Cardiol Clin. 1999;17:751-65.

2. Safi HJ, Miller CC III, Yawn DH, Iliopoulos DC, Subramaniam MH,
Harlin S, et al. Impact of distal aortic and visceral perfusion on liver function during thoracoabdominal and descending thoracic aortic repair. J Vasc Surg. 1998;27(1):145-52.

3. Griepp RB, Ergin MA. Aneurysms of the aortic arch. In: Edmunds LH, editor. Cardiac surgery in the adult. St Louis: Mosby; 1996. p. 1197-224.

\title{
Left atrial dissection after aortic valve replacement
}

\author{
Hiroshi Osawa, MD, PhD, Shinpei Yoshii, MD, PhD, Shigeru Hosaka, MD, PhD, \\ Shoji Suzuki, MD, PhD, Samuel J. K. Abraham, MD, PhD, and Yusuke Tada, MD, PhD, \\ Yamanashi, Japan
}

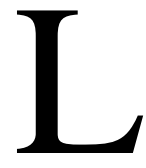

eft atrial dissection is a severe but rare complication occurring in $0.84 \%$ of mitral replacement cases. ${ }^{1}$

We report a case of left atrial dissection caused by aortic annular disruption after aortic valve replacement that was identified by means of transesophageal echocardiography and repaired immediately. Such a case has not been reported in the literature to our knowledge.

\section{Clinical Summary}

A 73-year-old woman underwent aortic valve replacement for aortic stenosis and concomitant coronary artery bypass grafting. Cardiopulmonary bypass was performed with moderate hypothermia and cardioplegic arrest. The left internal thoracic artery was anastomosed to the left anterior descending artery, and a saphenous vein graft was anastomosed to a branch of the obtuse marginal artery. After distal coronary bypass, an aortotomy was made. The aortic valve was found to be calcified, and the calcification extended to the aortic annulus. While removing the calcification, a dissection of the noncoronary annulus occurred and was repaired with everted mattress sutures, with which the prosthetic valve was also fixed. Then a Carbomedics 19-mm prosthetic valve (Sulzer Carbomedics, Austin, Tex) was implanted, and the aortotomy was closed. A proximal saphenous vein graft was anastomosed to the ascending aorta.

After successful weaning from cardiopulmonary bypass, a finding resembling dissection of the left atrium was found on routine intraoperative transesophageal echocardiography (Figure 1). Doppler echocardiography showed a flow originating from the aortic annulus and continuing into the atrial septal wall. The

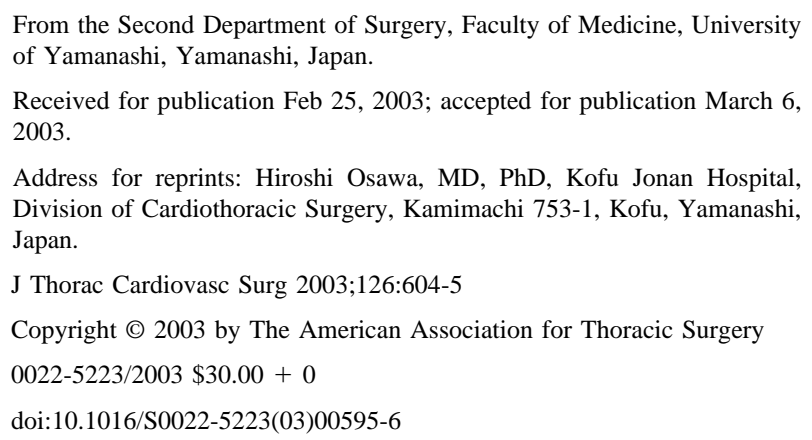

dissection cavity ruptured, and the blood was let out through a defect on the posterior side of the pericardium close to the inferior vena cava. However, the dissection cavity continued to fill up repetitively, and

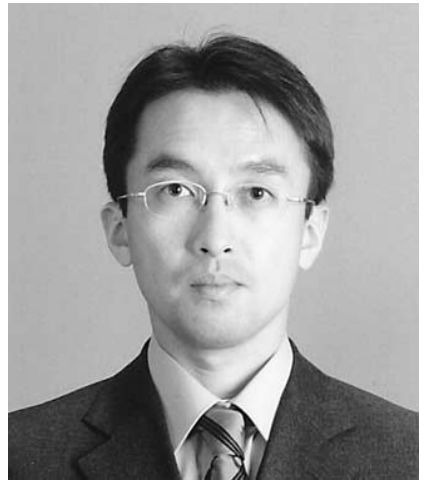

Dr Osawa hemodynamics worsened. We thought the entry might have been made by aortic annular disruption and resulted in dissection of the left atrium, as shown in Figure 2, A.

Cardiopulmonary bypass was reestablished, and an aortotomy was made again. When the prosthetic valve was removed, the noncoronary aortic annulus was divided, and entry into the left atrial dissection was made. The annulus, including the aortic wall, was reconstructed with a Hemashield Gold (Boston Scientific, Natick, Mass) Dacron patch, and aortic valve re-replacement was performed on the reconstructed annulus (Figure 2, B). The dissection cavity was decompressed, and cardiopulmonary bypass was successfully terminated. Postoperative echocardiography demonstrated no abnormal findings of the left atrial wall. The patient was discharged uneventfully, and she is doing well 3 years after the operation.

\section{Discussion}

Left atrial dissection is a rare complication and has been reported to occur in mitral valve surgery to an extent of $0.84 \%$. $^{1}$

In our case aortic annular disruption caused by the removal of annular calcification caused dissection of the left atrium. We examined a cadaver heart and found that division of the noncoronary aortic annulus allowed easy opening into the atrial septal wall, and the wall was comprised of 2 layers running in different directions. The above finding provides convincing evidence that an annular disruption in that site could easily pave the way for a dissection into the wall of the left atrium.

Left atrial dissection is a severe complication that results in low output syndrome and hemodynamic collapse. ${ }^{1,2}$ Most of the previously reported cases have required surgical treatment. ${ }^{1-4}$ It is necessary to close the entry of the dissection to treat this complication. In our case we had to reconstruct the destroyed annulus because the destroyed annulus could not withstand the replacement of the prosthetic valve again. We repaired the annulus with a 